\title{
Autoconcepto, expectativas y sentido de vida: Sinergia que determina el aprendizaje
}

\author{
Self-Concept, Expectations, and Meaning of Life: Sinergy That Determines Learning
}

Autoconceito, expectativas e sentido de vida: Sinergia que determina a aprendizagem

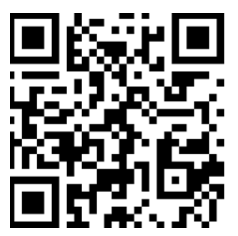

Corregido • Revised • Revisado: 01 / 10 / 2020

Aceptado • Accepted • Aprovado: 03 / 12 / 2020

Dora Esperanza Sevilla-Santo

Universidad Autónoma de Yucatán

Yucatán, México

dora.sevilla@correo.uady.mx

https://orcid.org/0000-0001-9781-6783

Mario José Martín-Pavón

Universidad Autónoma de Yucatán

Yucatán, México

mario.martin@correo.uady.mx

https://orcid.org/0000-0002-8458-137X

Sandra Paola Sunza-Chan

Universidad Autónoma de Yucatán

Yucatán, México

ssunza@correo.uady.mx

https://orcid.org/0000-0003-1964-8571

Nora Verónica Druet-Domínguez

Universidad Autónoma de Yucatán

Yucatán, México

ddoming@correo.uady.mx

https://orcid.org/0000-0002-3374-2620

\begin{abstract}
Resumen: El estudio buscó identificar los factores personales que se constituyen en barreras para el aprendizaje del estudiantado de las preparatorias estatales de Yucatán. Se desarrolló con el paradigma cuantitativo, con alcance correlacional. Las personas participantes fueron el estudiantado de primer año del ciclo escolar 2017-2018 (2599) y sus docentes (163). La recolección de información fue mediante un cuestionario aplicado al estudiantado, que indagó sobre su autoconcepto, expectativas, sentido de vida y antecedentes escolares; así como un guion para los grupos de enfoque con el profesorado, que identificó las barreras que se les presentan para lograr que sus estudiantes aprendan. El análisis de las relaciones se dio a través del coeficiente de correlación de Pearson, el estudio de los indicadores de cada factor y el análisis del discurso del profesorado. Los resultados señalan que entre las limitantes del aprendizaje se encuentran los bloqueos que el propio
\end{abstract}


http://doi.org/10.15359/ree.25-1.12

http://www.una.ac.cr/educare

educare@una.ac.cr

estudiantado erige, al no reconocer sus capacidades o desvalorarlas (autoconcepto negativo); así como su falta de metas hacia dónde dirigir sus acciones, propiciando un bajo compromiso al no encontrar sentido a los aprendizajes (sentido de vida). Esto evidencia fallas en la implementación del modelo educativo, al infravalorarse la formación para la vida en favor de la adquisición de conocimientos; se resalta la necesidad de formar al personal docente en la manera de retroalimentar, el reconocimiento de las potencialidades y en la aplicación de estrategias que formen para la vida; para que sea capaz de enriquecer el proceso formativo a partir de estos elementos.

Palabras claves: Autoconcepto; desempeño del estudiantado; educación media superior; expectativas.

Abstract: The study sought to identify the personal factors that constitute barriers to student learning in public high schools located in Yucatan. It was conducted under a quantitative paradigm, with a correlational scope. Participants were freshmen of the 2017-2018 school year (2599) and their teachers (163). The information was collected through a questionnaire applied to the students, who inquired about their self-concept, expectations, meaning of life, and school background. A script for the teacher's focus groups was also used; it identified the barriers they face to persuade the students to learn. The analysis of the relationships was obtained using Pearson's correlation coefficient, the study of indicators of each factor, and the analysis of the teachers' discourse. The results show that, among the limitations of learning, there are the blockades that the students form when they fail to recognize their capacities, or when they devalue them (negative self-concept), as well as their lack of goals to direct their actions, causing a low commitment as they don't find sense to the lessons (sense of life). This shows flaws in the implementation of the educational model by underestimating the training for life in favor of knowledge acquisition, highlighting the need to train the teaching staff in means of giving feedback, recognition of the student's potential, and application of strategies that prepare for life, so they can be able to enrich the formative process from these elements.

Keywords: self-concept, student's performance, high school education, expectations

Resumo: O estudo buscou identificar os fatores pessoais que constituem barreiras para a aprendizagem dos estudantes das preparatorias estatais de Yucatán. Se desenvolveu a través do paradigma quantitativo com alcance correlacional. Os participantes foram estudantes do primeiro ciclo escolar 2017-2018 (2599) e seus professores (163). A recoleção da informação foi um questionário aplicado aos estudantes que indagou sobre seu autoconceito, expetativas, sentido de vida e antecedentes escolares, assim como um guião para os grupos de enfoque com os professores que identificou as barreiras que apresentaram se para garantir que seus alunos aprendam. A análise das relações se deu através do coeficiente de correlação de Pearson, o estudo dos indicadores de cada fator e o análise do discurso dos professores. Os resultados sinalizam que entre que entre as limitantes de aprendizagem se encontram os bloqueios que os próprios estudantes nutrem ao não reconhecer suas capacidades ou as desvalorizar (autoconceito negativo) assim como falta de metas para dirigir suas ações propiciando um baixo compromisso ao não encontrar sentido aos aprendizados (sentido de vida). Isso evidência falhas na implementação do modelo educativo, ao subestimar a formação para a vida em favor da aquisição de conhecimentos, ressaltando se de formar os docentes em uma maneira de retroalimentar, os reconhecimento das potencialidades e na aplicação de estratégias que preparem para a vida, para que seja capaz de enriquecer o processo formativo a partir destes elementos.

Palavras chave: Autoconceito; desempenho do estudante; educação média superior; expetativas. 
http://doi.org/10.15359/ree.25-1.12

\section{Introducción}

El proceso de aprendizaje puede verse influido por un sinfín de factores, algunos de los cuales se originan en los contextos en los que el estudiantado se desarrolla cotidianamente, otros tienen relación con las propias instituciones educativas y algunos más dependen directamente del estudiantado; todo ello define el ritmo, estilo y alcance de lo que cada estudiante aprende. A estos factores, Booth y Ainscow (2002) los denominan barreras para el aprendizaje y la participación, al asumir que el hecho de que parte del estudiantado no aprenda a través de las formas tradicionales de enseñanza es una problemática de toda la comunidad educativa y no exclusivamente estudiantil.

Con esta perspectiva, Onrubia Goñi (2009, p. 51) afirma que "las barreras al aprendizaje y la participación se generan a través de la interacción entre los alumnos y sus contextos, entre las personas y las políticas, instituciones, culturas y circunstancias sociales y económicas que afectan a sus vidas"; de aquí que sea necesario evidenciarlas en cada contexto educativo, de tal forma que puedan ser atendidas para dar certidumbre a los procesos formativos.

Es importante resaltar que, aunque el concepto de barreras para el aprendizaje y la participación surge en el ámbito de la educación especial, con énfasis en las necesidades educativas que puede presentar el estudiantado (con una discapacidad o sin esta), actualmente los organismos internacionales como la Organización de las Naciones Unidas para la Educación, la Ciencia y la Cultura (UNESCO) (2017) promueven el reconocimiento de la diversidad que asiste a las aulas, la cual puede darse por características del estudiantado, del profesorado, de los modelos educativos y de los contextos en los que las escuelas se encuentran inmersas; y es responsabilidad de toda la comunidad educativa hacer visibles estas diferencias y enriquecer el proceso formativo con ellas.

De manera específica, en México se ha dado un giro en el sistema educativo estableciéndose políticas y lineamientos que buscan hacer realidad la atención a la diversidad en las aulas. En este sentido, la Dirección General de Educación Indígena (2012) señala:

En este contexto educación-sociedad-cultura, resulta imprescindible aprender a mirar la práctica educativa, reconociendo las formas de enseñar, los ritmos y estilos de aprender, las particularidades de los alumnos — las individuales, y las que son producto de su situación sociocultural- así como los diversos contextos en donde interactúan, revisando las aportaciones teóricas y prácticas que se realizan con respecto a la educación desde diversas visiones. (p. 13)

A partir de lo referido anteriormente se hace necesario analizar los factores que pueden constituirse en barreras para el aprendizaje que surgen de la diversidad descrita, por lo que el estudio del cual deriva el presente artículo buscó analizar los factores personales e institucionales. Sin embargo, desde la perspectiva del equipo de autoría del presente artículo, el punto de 
http://doi.org/10.15359/ree.25-1.12

http://www.una.ac.cr/educare

educare@una.ac.cr

partida debe ser el propio estudiantado y sus características; ya que identificadas se podrán plantear estrategias de atención que los diferentes programas y servicios implementados en las escuelas (como los programas de tutoría y orientación, entre otros), puedan abordar en los espacios disponibles para ello. De aquí que este documento solo haga referencia a estos últimos.

Dentro de los factores personales que pueden llegar a constituirse en barreras para el aprendizaje, la bibliografía reporta: el sexo, la edad, los antecedentes académicos, los conocimientos previos, la motivación, la autoestima, el desarrollo social y emocional, la personalidad, las estrategias de aprendizaje y las expectativas, la madurez cognitiva, las habilidades metacognitivas, el pensamiento crítico, el autoconcepto, el sentido de vida, entre otros muchos (Yuen et al., 2017); de cuya incidencia depende del contexto y nivel educativo en el que se estudian.

Ante esta diversidad de factores que pueden constituirse en barreras para el aprendizaje del estudiantado, una reflexión del presente artículo permitió enfocar el análisis en tres de estos: autoconcepto, expectativas y sentido de vida; dado que se relacionan directa o indirectamente con los otros reportados y porque el déficit de ellos puede desencadenar una serie de problemáticas académicas y sociales.

Al respecto, Abramoski et al. (2018) señalan que la falta de consolidación en el desarrollo de este tipo de factores en adolescentes se relaciona con el consumo de alcohol o drogas, depresión, delincuencia, embarazos no deseados entre otras situaciones que constituyen algunas de las razones de la reprobación, abandono o rezago escolar. Hecho que sustenta la idea de que estos factores pueden tener un efecto protector contra conductas de riesgo, si se desarrollan apropiadamente, ya que son menos atractivas para aquellas personas cuyas metas están bien definidas, característica de quienes cuentan con un autoconcepto positivo, altas expectativas y un sentido de vida.

\section{Planteamiento del problema}

De acuerdo con información presentada por la Organización para la Cooperación y el Desarrollo Económicos (OCDE, 2017), solamente el 12\% de jóvenes de los países de este organismo concluyen la educación media superior; asimismo, en América Latina el 37\% de adolescentes de entre 15 y 19 años abandonan este nivel educativo. En la misma línea, el Sistema de Información de Tendencias Educativas de América Latina (SITEAL) (2010) reporta que, en 2009, menos del 50\% de jóvenes de 20 años consiguió terminar el bachillerato.

En cuanto a México, Gámez Rojas (2018) presenta un análisis de la eficiencia terminal y su relación con la deserción y la reprobación en las cohortes de 1990 a 2012, encontró que solo se ha avanzado 7 puntos desde entonces pasando de una eficiencia terminal de $55,2 \%$ a 61,8\%. De igual manera, el estudio desarrollado por Miranda López (2018) señala que la tasa de aprobación en bachillerato, en el ciclo escolar 2014-2015, fue de tan solo el 84,4\%. Asimismo, 
http://doi.org/10.15359/ree.25-1.12

refiere que el porcentaje de estudiantes con extraedad grave, para el ciclo escolar siguiente, fue de 13,5\%, lo que da cuenta de las dificultades del alumnado en su tránsito por este nivel educativo; relaciona dichas problemáticas con la precariedad de las instituciones en la que el estudiantado se encuentra matriculado.

En este mismo sentido, de acuerdo con el Instituto Nacional para la Evaluación de la Educación (INEE) (2012), el 15,9\% de jóvenes abandona sus estudios de bachillerato, nivel educativo en donde se presenta el mayor rezago educativo (7,3\%). Así mismo, este organismo señala que en 2015 fue este nivel educativo el que presentó el porcentaje más alto de alumnado con extraedad grave (14,9\%) con una eficiencia terminal de tan solo el 63\%. Particularmente en Yucatán, el porcentaje de deserción es superior a la media nacional al ubicarse en 17,8\% (en el ciclo escolar 2013-2014) con un porcentaje de eficiencia terminal del 57,5\%.

Esta problemática ha motivado el desarrollo de estudios que han tratado de identificar los factores que limitan el aprendizaje del estudiantado. Se reporta, de manera consistente, la falta de visualización del estudiantado de que culminar los estudios de bachillerato y realizar estudios de nivel superior puede ser un medio para tener una mejor calidad de vida. Ejemplo de esto es el estudio realizado por Pereyra López y Padilla González (2013), que buscó identificar los elementos de las prácticas escolares que inciden de manera positiva en el desempeño del estudiantado, así como el análisis de los factores personales e institucionales que afectan su trayectoria.

Estos datos resaltan la necesidad de identificar los factores personales que se constituyen en barreras para el aprendizaje estudiantil y que propician el bajo rendimiento académico, la reprobación y la deserción escolar, tal como lo refieren Ruiz-Ramírez et al. (2014), con la intención de establecer estrategias que ayuden a revertir los efectos nocivos de estos.

Por lo anterior, el objetivo del presente estudio es identificar los factores personales que se constituyen en barreras para el aprendizaje del estudiantado de primer año de las escuelas preparatorias estatales de Yucatán.

\section{Referentes conceptuales}

Los factores personales que pueden llegar a ser obstáculo para que el estudiantado avance en su proceso formativo han sido agrupados para su estudio en cognitivos (pensamiento reflexivo, hábitos de estudio, antecedentes académicos, etc.) y no cognitivos (motivación, autoconcepto, expectativas, etc.); ambos tipos pueden ser atendidos desde las instituciones educativas a través de diferentes estrategias (Martín Pavón, 2015).

Dentro del amplio espectro de factores que tienen un impacto determinante en el aprendizaje, se encuentra el autoconcepto, definido por García y Musitu (2001) como un constructo que representa la concepción que la persona tiene de sí misma como ser físico, social y espiritual. Es decir, implica una serie de creencias positivas y negativas que llevan a una autoevaluación. 
http://doi.org/10.15359/ree.25-1.12

http://www.una.ac.cr/educare

educare@una.ac.cr

Al respecto de este factor, estudios como los de Goñi Palacios y Fernández Zabala (2007) enfatizan que no puede entenderse la conducta del estudiantado en la escuela, si no se consideran las percepciones que tiene de sí mismo y de su competencia académica. Esta imagen puede ir cambiando, dependiendo del contexto en el que se desenvuelve la persona (casa, escuela, iglesia, trabajo, etc.) y del momento que esté viviendo. De aquí su relevancia en el ámbito educativo, ya que, si el estudiantado no se percibe como capaz de realizar las actividades implicadas en el proceso formativo, podrá bloquearse o no plantearse metas por considerar que no las alcanzará; por tanto, en la medida en la que el estudiantado se sienta bien consigo mismo y confíe en sus capacidades, podrá hacer frente a las demandas y retos que le suponga el aprender.

Un problema presente, en este sentido, es que, como parte de los procesos formativos desarrollados en las instituciones educativas de los diferentes niveles, tanto profesorado como familiares han dado un peso determinante a lo que el estudiantado no puede hacer; es decir, se resaltan sus fallas y limitaciones, y pocas veces se orienta la mirada a sus fortalezas y capacidades (Sevilla Santo et al., 2020).

De manera particular, durante la adolescencia, el estudiantado se enfrenta a una serie de cambios académicos que se suscitan de forma rápida: a los 12 años ingresa a secundaria, a los 15 a bachillerato y a los 18 a la educación superior (aproximadamente); cada uno de estos niveles les demanda más competencias, sobre todo cognitivas. Además, se relaciona con una variedad de profesorado del que recibe una retroalimentación constante y diversa (Santana Vega y García, 2011); y también tiene relación con nuevos compañeros y compañeras, quienes, la mayoría de las veces, servirán para compararse e irse definiendo.

Al respecto, Ibarra Aguirre y Jacobo García (2016) señalan que "en la medida que los adolescentes se van empoderando cognitivamente y toman conciencia de sus competencias académicas" (p. 61), irán influyendo en la definición que hagan de sí en todos los ámbitos de su vida y de forma puntual en su desarrollo académico en la escuela.

La importancia de este factor ha motivado el desarrollo de una serie de estudios en el ámbito escolar vinculándolo con variables como la motivación, el rendimiento académico, las estrategias de aprendizaje y las dificultades de aprendizaje (Costa y Tabernero, 2012).

Entre las estrategias que se proponen para el desarrollo de este factor, algunos estudios mencionan que la actividad física favorece el desarrollo de un autoconcepto positivo, lo cual tendrá una repercusión directa en el rendimiento académico (Chacón Borrego et al., 2017). Otros afirman que es posible desarrollarlo a partir de las interacciones que el estudiantado establece con otros actores educativos y de las experiencias escolares que vaya viviendo (Goñi Palacios, 2009). Esto debe dar la clave para que las instituciones educativas capaciten al profesorado sobre la forma de orientar y retroalimentar el proceso de aprendizaje del estudiantado; incluyan en los programas de tutorías actividades que contribuyan al desarrollo del autoconcepto; así 
http://doi.org/10.15359/ree.25-1.12

como que fomenten en las aulas el trabajo colaborativo más que el competitivo; y reconozcan la diversidad que existe y enriquezcan el proceso educativo con ella.

Otro factor vinculado con el aprendizaje y el desempeño académico del estudiantado es el de las expectativas. En el caso del bachillerato, estas se encuentran relacionadas con su interés por continuar sus estudios hacia niveles superiores y con sus metas académicas. En este sentido, la (OECD, 2019) revela que, en México, el tener un título profesional permite a los grupos egresados participar más en el mercado laboral, con mejores resultados de empleo y entre estos, contar con mejores salarios que quienes no llegan a este nivel educativo; de aquí que la sociedad pondere, de forma significativa, el que sus jóvenes realicen estudios de nivel superior.

De acuerdo con Sánchez-Sandoval y Verdugo (2016), la conceptualización de expectativas a futuro se refiere a la medida en que un individuo espera que ocurra un evento, lo cual lo lleva a establecer objetivos y planear acciones. Esta tendencia, si bien está presente a lo largo de toda la vida, adquiere una especial importancia en la adolescencia, debido a que las exigencias de preparación para la vida adulta convierten la formulación de un proyecto de vida en una de las tareas evolutivas más relevantes de esta etapa; pues las expectativas y metas están determinadas por varios factores, entre ellos, los valores de los padres y madres respecto del logro académico, el género y las propias expectativas del profesorado hacia el alumnado (Papalia et al., 2009).

Además, los grupos adolescentes se encuentran en una etapa donde existe mucha incertidumbre por la dificultad de hallar los medios y herramientas para consolidar sus expectativas y metas, lo cual también influye en la definición de estas. En este sentido, Schwinger y Wild (2012) consideran que en los contextos educativos es pertinente una visión más centrada en el estudiantado, dado que se relaciona de modo directo con las creencias de autoeficacia y autoconcepto académico, pues tienen un valor predictivo muy relevante sobre la implicación del estudiantado en su proceso de aprendizaje y, por ende, sobre sus logros académicos. Es decir, si un alumno o alumna tiene una percepción positiva de sí y de sus capacidades, se planteará metas más altas y se encontrará más implicado en alcanzarlas.

Lo anterior se encuentra relacionado con las ideas de Calderón y Casu (2011), quienes señalan que si las expectativas del estudiantado están orientadas al aprendizaje, su conducta será más adaptativa con respecto a las dificultades que puedan presentar las tareas que deba realizar para alcanzar sus metas, lo cual afectará, de modo positivo, su rendimiento y persistencia en los estudios.

Con base en las ideas anteriormente analizadas, puede señalarse que así como las expectativas hacia el estudio y las metas académicas del estudiantado pueden ser aspectos que influyan de modo positivo en su aprendizaje y éxito escolar, si estas expectativas y metas son bajas o no se encuentran claramente planteadas, pueden constituirse en barreras para el aprendizaje, debido a que la falta de un proyecto de vida es uno de los factores que influye en el bajo desempeño académico (Gualtero Pinzón, 2016). 
http://doi.org/10.15359/ree.25-1.12

http://www.una.ac.cr/educare

educare@una.ac.cr

En relación con el proyecto de vida, es importante señalar que este se construye a partir de tener un sentido de vida. De acuerdo con Frankl (1991), quien acuñó este término, "la primera fuerza motivante del hombre es la lucha por encontrarle un sentido a su propia vida" (p. 100). Esta búsqueda lleva al individuo a actuar de determinada forma ante las distintas situaciones que se le presentan, lo dirige a un objetivo o meta que va más allá de él mismo, donde los contextos escolares juegan un papel importante en la definición del sentido de vida (Kashdam y McKnight, 2009).

Con esta perspectiva, de manera específica, Bruzzone (2008) señala que la educación desempeña un papel importante en la formación de adolescentes, al tener como valor prioritario el desarrollo del potencial humano y de ayudarles a crecer como personas para que aprendan a estar en el mundo de manera autónoma; para lo cual se requiere de una educación centrada en el desarrollo de un sentido de vida.

Sin embargo, de Barbieri (2018) refiere que, a pesar de todos los planteamientos y acciones que se han llevado a cabo en la educación, estos no han sido suficientes, puesto que en las escuelas se sigue priorizando el desarrollo de aptitudes para el saber conocer y el saber hacer; no apoyando a sus estudiantes a encontrar un sentido al aprendizaje.

Por otra parte, Moreno Carmona y Rodríguez Fernández (2010, p. 9) manifiestan que "el bienestar podría estar vinculado a la consecución de planes personales, objetivos y proyectos vitales, muy relacionados a su vez con el propósito o Sentido de la vida". Si esto se ubica en el contexto escolar, se puede asumir que aquel alumnado con un sentido en su vida tendrá metas objetivas y realistas vinculadas a su formación académica, por lo que la probabilidad de alcanzar estas es mayor a la de quienes carecen de este.

Como puede verse, el sentido de vida es un factor de protección, especialmente en la adolescencia y su falta de desarrollo puede abrumar, debido a que puede ser difícil vislumbrar su futuro si no se tienen objetivos claros hacia los cuales dirigir las acciones de su vida.

\section{Método}

\section{Tipo de estudio}

El estudio del cual deriva el presente artículo se desarrolló desde el paradigma cuantitativo, de carácter mixto puesto que se usaron técnicas de recolección y análisis tanto cuantitativas como cualitativas. En relación con su alcance, este corresponde al de un estudio correlacional, ya que se buscó establecer la influencia de los factores personales estudiados en el aprendizaje del estudiantado, caracterizado por su rendimiento académico. De acuerdo con la temporalidad de la recolección y análisis de la información, este clasifica como un estudio transeccional ya que dichas actividades se realizaron en un solo momento (Cohen et al., 2011). 
http://doi.org/10.15359/ree.25-1.12

http://www.una.ac.cr/educare educare@una.ac.cr

\section{Participantes}

La población en estudio estuvo conformada por todo el estudiantado de primer año del Subsistemas de Escuelas Preparatorias Estatales de Yucatán, inscritos al ciclo escolar 2017-2018 $(\mathrm{N}=3032)$; así como el profesorado que le impartió clases $(\mathrm{N}=425)$. Para el caso del estudiantado, la muestra se integró a través de un censo, es decir, se encuestó a todo el estudiantado de primer año que estuvo presente al momento de la aplicación (2599). De igual forma, para el caso del profesorado se consideró a quienes se encontraban en los centros educativos al momento de realizar los grupos de enfoque (163).

\section{Instrumentos}

\section{Cuestionario para estudiantes}

Para indagar sobre el autoconcepto, las expectativas y el sentido de vida, se construyó un instrumento dirigido al estudiantado, a partir de la revisión de la bibliografía y el contexto del subsistema de Escuelas Preparatorias Estatales. Para el caso particular del sentido de vida, se tomó como base la versión en español del Purpose in Life (Propósito de vida), de Noblejas de la Flor (1994).

Integrada la versión preliminar, se realizó un piloteo aplicándolo a un total de 449 estudiantes de primer año de una escuela preparatoria con las mismas características que las pertenecientes al Subsistema de Escuelas Preparatorias Estatales; se generó una base de datos en el paquete estadístico Statistics Package for Social Science (SPSS, v. 21).

El análisis realizado con la información obtenida a través del estudio piloto incluyó una prueba de discriminación de los reactivos, comparación delas puntuaciones del estudiantado que obtuvo una puntuación superior al cuartil 75, con la de estudiantes cuya puntuación fue inferior al cuartil 25; esta comparación se realizó a través de la prueba t para muestras independientes. El resultado arrojó que de los 67 reactivos de la versión preliminar del instrumento solamente discriminaron 52.

Adicionalmente, con la intención de revelar las dimensiones verdaderas en las que se agrupan los reactivos, se realizó un análisis de factores por el método de componentes principales y la rotación Varimax; se acordó considerar como factores aquellos que agruparan al menos tres ítems y tuvieran cargas factoriales mayores o iguales a 0,40. El resultado de este análisis indicó la conformación de tres factores. La versión final del instrumento quedó compuesta de 38 ítems.

Las definiciones operacionales de dichos factores estudiados se presentan a continuación:

Autoconcepto: Valoración que estudiantes hacen de su capacidad para realizar las actividades académicas que se les encarga y la posesión de atributos que contribuyen a su buen desempeño escolar (14 ítems). 
http://doi.org/10.15359/ree.25-1.12

http://www.una.ac.cr/educare

educare@una.ac.cr

Expectativas: Refiere lo que estudiantes esperan lograr como consecuencia de la conclusión de sus estudios, tanto en el ámbito académico como personal (4 ítems).

Sentido de vida: Intención estable y generalizada para lograr algo que es significativo para la persona y que impacta en su mundo (20 ítems).

En cuanto a la confiabilidad del instrumento, esta se calculó a través del alfa de Cronbach. Su valor de 0,955 lo hace un instrumento confiable.

\section{Guion de entrevista para profesorado}

La recolección de datos con el profesorado se realizó a través de grupos de enfoque, para lo que se construyó un guion de entrevista que indagó sobre cuáles son las principales barreras que se les presentan para lograr que sus estudiantes se apropien de los conocimientos de sus asignaturas, la manera como las han enfrentado y los requerimientos para superarlas. Este se integró con cuatro preguntas abiertas que propiciaron la discusión sobre los aspectos mencionados.

\section{Análisis de la información}

Para identificar cuáles de los factores estudiados podrían constituirse en barreras para el aprendizaje, se construyeron indicadores en escala de cero a cien para cada uno de ellos, los cuales se definen en función de las puntuaciones mínimas y máximas posibles de alcanzar por los sujetos encuestados en cada uno de los apartados correspondientes a estos. Dichos indicadores se conciben como el cociente entre la diferencia de la suma de los reactivos con el valor mínimo y el rango, multiplicándose dicho resultado por cien. Así, el indicador del factor resulta del promedio de las cantidades obtenidas para cada estudiante a través de la Fórmula 1:

Fórmula 1

$$
\mathrm{I}_{\mathrm{F}}=\left(\frac{\mathrm{S}_{\mathrm{I}}-\mathrm{Min}}{\text { Rango }}\right) \times 100
$$

Donde:

$I_{F}=$ Indicador del factor en estudio.

$S_{I}=$ Suma de los reactivos del instrumento que miden el factor estudiado.

Min = Puntaje mínimo posible en el apartado del factor estudiado.

Rango $=$ Diferencia entre los puntajes máximo y mínimo del apartado del factor estudiado. 
http://doi.org/10.15359/ree.25-1.12

Asimismo, se construyó un indicador del aprendizaje, caracterizándolo a través del rendimiento académico del estudiantado a partir de la Fórmula 2:

Fórmula 2

$$
I R=\frac{P B+100 * P S}{2}+\operatorname{RepS}+\operatorname{RepB}-\text { Mrep }
$$

Donde:

$I R=$ Indicador de rendimiento académico

$P B=$ Promedio de las calificaciones de primer semestre de preparatoria

$P S=$ Promedio general de secundaria

RepS = Hecho de que el alumnado reprobara o no algún grado de secundaria ( $1=$ No y $0=\mathrm{Si})$

$\operatorname{Rep} B=$ Hecho de que el alumnado reprobara o no algún grado de bachillerato ( $1=$ No y $0=\mathrm{Si})$

Mrep $=$ Número de asignaturas reprobadas en secundaria.

Posteriormente, se analizó la relación entre los factores estudiados y el aprendizaje del estudiantado, correlacionando las puntuaciones de los indicadores respectivos mediante el coeficiente de correlación de Pearson.

También se clasificó al estudiantado de acuerdo con el nivel de autoconcepto, expectativas y estado de sentido de vida en relación con su puntuación en los indicadores respectivos, tal y como se describe a continuación: Menos de 70 puntos (bajo autoconcepto, bajas expectativas y vacío existencial), entre 70 y 85 puntos (autoconcepto medio, expectativas moderadas e incertidumbre) y mayor a 85 puntos (alto autoconcepto, altas expectativas y sentido de vida); se acordó que un factor se constituiría en barrera para el aprendizaje del estudiantado, si guardaba relación con el aprendizaje y al menos el 30\% de los sujetos encuestados se encontraba en los niveles de bajo autoconcepto, bajas expectativas o vacío existencial.

La información obtenida a partir de las entrevistas grupales con el profesorado fue reunida en categorías para su análisis. Dichas categorías surgieron de la revisión de las transcripciones y el contraste con lo reportado en la literatura consultada; así como el apego a los objetivos del estudio. Sin embargo, también se identificaron en este proceso otras categorías que se incorporaron al tratamiento de los datos.

\section{Resultados}

Un primer análisis realizado para conocer el contexto en el que se desarrolló el estudio permitió determinar que el 92,2\% del estudiantado cursó la secundaria en una escuela pública; 
http://doi.org/10.15359/ree.25-1.12

http://www.una.ac.cr/educare

educare@una.ac.cr

que el 4,8\% manifestó haber reprobado algún grado de este nivel educativo. Asimismo, se determinó que el promedio de materias reprobadas en secundaria fue de 2,3 y que el 6,5\% está recursando el primer grado de bachillerato.

Para identificar los factores personales que se constituyen en barreras para el aprendizaje del estudiantado en cada una de las escuelas, con la información recabada en el cuestionario aplicado a este colectivo, se estudió la relación entre estos y el indicador de aprendizaje a través del coeficiente de correlación de Pearson; en la Tabla 1 se presentan los resultados para el factor autoconcepto.

Tabla 1: Análisis del autoconcepto como posible barrera para el aprendizaje

\begin{tabular}{ccc}
\hline Escuela & $r$ & $p$ \\
\hline 1 & 0.218 & 0.002 \\
2 & 0.231 & 0.001 \\
3 & 0.083 & 0.064 \\
4 & 0.288 & 0.001 \\
5 & 0.286 & 0.002 \\
6 & 0.323 & 0.001 \\
7 & 0.290 & 0.001 \\
8 & 0.228 & 0.003 \\
9 & 0.227 & 0.015 \\
10 & 0.234 & 0.002 \\
11 & 0.421 & 0.001 \\
12 & 0.328 & 0.014 \\
\hline
\end{tabular}

Nota: Elaboración propia.

Del análisis de la Tabla 1 se observa que la única escuela en donde el autoconcepto no influye en el aprendizaje del estudiantado es en la número 3.

Adicionalmente, en la Tabla 2 se presenta la clasificación por nivel de desarrollo del factor autoconcepto en cada escuela.

En la Tabla 2 se observa que más del $40 \%$ del estudiantado de cada escuela presenta un nivel de desarrollo del autoconcepto bajo, incluso en la escuela 3 en la que dicho factor no guardó relación con el aprendizaje del estudiantado; sin embargo, de acuerdo con lo señalado en la metodología, este factor no se constituye en una barrera para el aprendizaje en dicha escuela. 
Tabla 2: Nivel de desarrollo del autoconcepto por escuela

\begin{tabular}{cccc}
\hline \multirow{2}{*}{ Escuela } & \multicolumn{3}{c}{ Nivel } \\
\cline { 2 - 4 } & Alto autoconcepto & Autoconcepto medio & Bajo autoconcepto \\
\hline 1 & 19.5 & 22.1 & 58.4 \\
2 & 24.3 & 22.0 & 53.7 \\
3 & 14.2 & 25.7 & 60.1 \\
4 & 27.8 & 27.8 & 44.4 \\
5 & 16.5 & 20.9 & 62.6 \\
6 & 20.4 & 21.3 & 58.3 \\
7 & 20.1 & 23.1 & 56.8 \\
8 & 25.6 & 27.3 & 47.1 \\
9 & 33.1 & 26.1 & 40.8 \\
10 & 16.6 & 29.3 & 54.1 \\
11 & 21.5 & 32.9 & 45.6 \\
12 & 27.1 & 11.9 & 61.0 \\
\hline
\end{tabular}

Nota: Elaboración propia.

Al respecto del factor expectativas, en la Tabla 3 se presenta el análisis de este factor como posible barrera para el aprendizaje del estudiantado.

Tabla 3: Análisis de las expectativas como posible barrera para el aprendizaje

\begin{tabular}{ccc}
\hline Escuela & $r$ & $p$ \\
\hline 1 & 0.101 & 0.163 \\
2 & 0.163 & 0.022 \\
3 & 0.048 & 0.291 \\
4 & 0.271 & 0.001 \\
5 & 0.128 & 0.175 \\
6 & 0.249 & 0.001 \\
7 & 0.132 & 0.071 \\
8 & 0.086 & 0.102 \\
9 & 0.165 & 0.080 \\
10 & 0.129 & 0.087 \\
11 & 0.046 & 0.692 \\
12 & 0.367 & 0.005 \\
\hline
\end{tabular}

Nota: Elaboración propia. 
http://doi.org/10.15359/ree.25-1.12

http://www.una.ac.cr/educare

educare@una.ac.cr

A partir del análisis de la Tabla 3 se puede identificar que solamente en las escuelas 2, 4, 6 y 12 las expectativas influyen en el aprendizaje del estudiantado.

En relación con el nivel de las expectativas, en la Tabla 4 se presenta la clasificación por nivel de estas en cada escuela.

Tabla 4: Nivel de expectativas por escuela

\begin{tabular}{cccc}
\hline \multirow{2}{*}{ Escuela } & \multicolumn{3}{c}{ Nivel } \\
\cline { 2 - 4 } & Altas expectativas & Expectativas moderadas & Bajas expectativas \\
\hline 1 & 51.3 & 25.1 & 23.6 \\
3 & 57.1 & 18.9 & 24.0 \\
4 & 58.1 & 22.1 & 19.8 \\
5 & 57.2 & 29.4 & 13.4 \\
6 & 47.8 & 22.6 & 29.6 \\
7 & 71.6 & 17.5 & 10.9 \\
8 & 58.3 & 16.6 & 25.1 \\
9 & 67.4 & 18.1 & 14.5 \\
10 & 62.7 & 25.3 & 12.0 \\
11 & 58.0 & 21.0 & 21.0 \\
12 & 59.5 & 24.1 & 16.4 \\
\hline
\end{tabular}

Nota: Elaboración propia.

Del análisis de la Tabla 4 se observa que menos del $30 \%$ del estudiantado de cada escuela tiene bajas expectativas, por lo que este factor no se constituye en una barrera para el aprendizaje en ninguna de ellas.

En cuanto al factor sentido de vida, en la Tabla 5 se presenta el estudio de la relación de este con el aprendizaje del estudiantado.

En la Tabla 5 se observa que las únicas escuelas en las que el sentido de vida no se relaciona con el aprendizaje son la 5, 11 y 12. 
http://doi.org/10.15359/ree.25-1.12

http://www.una.ac.cr/educare educare@una.ac.cr

Tabla 5: Análisis del sentido de vida como posible barrera para el aprendizaje

\begin{tabular}{ccc}
\hline Escuela & $r$ & $p$ \\
\hline 1 & 0.188 & 0.020 \\
2 & 0.318 & 0.001 \\
3 & 0.223 & 0.001 \\
4 & 0.343 & 0.001 \\
5 & 0.171 & 0.196 \\
6 & 0.267 & 0.001 \\
7 & 0.184 & 0.024 \\
8 & 0.195 & 0.001 \\
9 & 0.352 & 0.001 \\
10 & 0.136 & 0.036 \\
11 & 0.043 & 0.712 \\
12 & 0.126 & 0.394 \\
\hline
\end{tabular}

Nota: Elaboración propia.

En cuanto al estado del sentido de vida, en la Tabla 6 se presenta la clasificación por escuela.

Tabla 6: Estado del sentido de vida por escuela

\begin{tabular}{cccc}
\hline \multirow{2}{*}{ Escuela } & \multicolumn{3}{c}{ Estado } \\
\cline { 2 - 4 } & Sentido de vida & Incertidumbre & Vacío existencial \\
\hline 1 & 14.4 & 29.3 & 56.3 \\
2 & 9.4 & 38.5 & 52.1 \\
3 & 9.1 & 39.6 & 51.3 \\
4 & 8.7 & 46.5 & 44.8 \\
5 & 11.2 & 35.5 & 53.3 \\
6 & 13.0 & 45.7 & 41.3 \\
7 & 8.4 & 34.1 & 57.5 \\
8 & 9.6 & 37.9 & 52.5 \\
9 & 18.0 & 54.0 & 28.0 \\
10 & 10.0 & 42.5 & 47.5 \\
11 & 14.1 & 51.3 & 34.6 \\
12 & 21.2 & 34.6 & 44.2 \\
\hline
\end{tabular}

Nota: Elaboración propia. 
http://doi.org/10.15359/ree.25-1.12

http://www.una.ac.cr/educare

educare@una.ac.cr

A partir de la Tabla 6 se evidencia que la única escuela en donde el porcentaje de estudiantes que se ubica en el nivel de vacío existencial es menor al 30\% es en la 9, por lo que este factor no se constituye en una barrera para el aprendizaje del estudiantado en ella.

Con la intención de complementar el análisis realizado con la información proporcionada por el estudiantado en el cuestionario, se realizaron grupos de enfoque con el profesorado de primer grado de cada una de las escuelas; a través de los cuales se identificaron una serie de factores personales, sociales e institucionales que el profesorado reporta como barreras para el aprendizaje. No obstante, para efectos de este artículo solo se dará cuenta de los factores personales identificados, siendo que en las viñetas presentadas se representa la opinión de un profesor $(P)$ de cada una de las escuelas participantes (esc) a manera de ejemplo de lo dicho por estos.

Un comentario constante del profesorado, en la mayoría de las escuelas, hace referencia a que el propio alumnado pone límites a su aprendizaje, al considerar que no son capaces de realizar las tareas que se les encargan y conformarse con calificaciones mínimas; además de que sienten inseguridad de sus capacidades y tienen temor a equivocarse. Estos argumentos toman sustento en los siguientes comentarios:

... los alumnos se ponen límites y se ponen barreras ellos solitos en cuanto al desempeño; o sea, con la calificación mínima están contentos, no buscan hacer un esfuerzo más. (P.1, esc. 10)

A los alumnos les da vergüenza o miedo a decir, a hablar, a expresarse; con temor a lo mejor de que esté mal lo que están diciendo; con temor a que el compañero de a lado se burle, no lo sé, pero ese temor, ese miedo a equivocarse, que creo que es la palabra correcta..., creo que es la barrera más grande que hay... (P.3, esc.1)

Como puede apreciarse, el estudiantado no reconoce sus capacidades y habilidades, lo cual limita su participación en clase y, al mismo tiempo, no permite aclarar sus dudas. Esta situación desencadena un rezago en la apropiación de los aprendizajes esperados en las asignaturas propiciando un bajo rendimiento académico, lo que en algunos casos puede terminar incluso en la deserción escolar; hecho que concuerda con lo encontrado a través de la información proporcionada por el estudiantado. De aquí que trabajar el autoconcepto académico sea una de las tareas prioritarias para los centros educativos del subsistema en estudio.

También el profesorado señala que el alumnado no se percata de la importancia de cursar el bachillerato y de las implicaciones que este nivel educativo tiene para su futuro; así como de la relevancia de su formación para su incorporación a la educación superior, haciendo el mínimo esfuerzo para acreditar las asignaturas. Hecho que habla de una falta de expectativas, pero también de un estado de su sentido de vida inapropiado; situación que reportan presente en un porcentaje importante de estudiantes, lo que complica la labor del personal docente para hacer que se apropien de los contenidos. Esta afirmación toma sustento en los siguientes comentarios: 
http://doi.org/10.15359/ree.25-1.12

Los alumnos tienen un desánimo increíble, de hecho, le pudiera decir yo sin temor a equivocarme, que en 24 años que llevo trabajando acá, es el primer año que me he encontrado con el índice más alto de desinterés por parte de los alumnos. No sé qué les pasa, les preguntas a qué vienen a la escuela y te dicen que porque los mandan o hacer amigos. (P.5, esc. 3)

También veo que los muchachos están muy desorientados, no saben lo que quieren estudiary eso hace que no valoren lo que se les enseña, algunos con los que he hablado un poco más me han dicho que quieren solamente buscar un trabajo de lo que sea. (P.7, esc. 8)

La cultura de no al esfuerzo, o sea los muchachos no quieren hacer realmente nada, ese es un problema de ambiente, conductual. Parece que más del $50 \%$ de los alumnos vienen obligados a clase, vienen porque tienen que venir, los manda su familia, pero no sé, los chicos no están preparados para la escuela porque están obligados y por venir obligado se desquitan con nosotros, entran al salón y no están poniendo atención ... se están durmiendo en el salón. (P. 2, esc. 12)

Aunado a lo anterior, el profesorado comenta que el alumnado no ve su educación como una prioridad, algunos o algunas solo van a la preparatoria para salir de casa y estar con gente de su edad, por lo que para estos grupos no es prioridad aprender; afirmación que se sustenta en los siguientes comentarios:

También creo que los alumnos tienen la idea de trabajar por su propia cuenta. Hubo una situación con un alumno de "¿oye por qué no me entregas tareas? ¿Por qué estás ausente en mi clase?" Solo vienen para darles a sus papás un papel de que cumplieron con la preparatoria, porque consideran que estudiar no les va dar de comer... (P. 4, esc. 2)

... ellos no lo ven ya como parte de su formación profesional, no lo ven como para tener un nivel de vida de mayor amplitud, ellos lo ven como que no van a comer de un certificado de preparatoria, o sea ellos creen que van a generar dinero por su propia cuenta. (P.2, esc. 5)

Como puede verse, contrario a la información proporcionada por el estudiantado, desde la perspectiva del equipo de docentes las bajas expectativas que tienen sus estudiantes limita su aprendizaje; ya que no son capaces de visualizarse en un futuro inmediato, ni de valorar su formación académica para su incorporación a los estudios de nivel superior o al mercado laboral, lo cual propicia un desinterés por culminar el bachillerato.

De manera más específica, el profesorado refiere la falta de un proyecto de vida. Sobre este punto señalan que el alumnado no saben qué es lo que quiere para su futuro, porque cuando se le cuestiona al respecto no sabe qué responder; además de que una parte toma decisiones acerca de su futuro con base en lo que está de "moda" o lo que consideran que es mejor pagado, como lo evidencian los siguientes comentarios: 
http://doi.org/10.15359/ree.25-1.12

http://www.una.ac.cr/educare

educare@una.ac.cr

Yo creo, y ya ahorita reflexionando, la semana pasada tuve una experiencia con primer año, es replantearnos realmente ¿qué es lo que los motiva? O sea, realmente ¿por qué vienen a la escuela? O sea, me he topado con veces en las que me dicen: "no sé.". Sí pero, ¿y qué?... Digo: "¿qué te motiva a ti?" A veces les preguntas y te dicen: no, yo quiero ser abogado, y cuando les preguntas ¿por qué? En esa parte es donde se bloquean. (P.6, esc. 4)

La semana pasada, platicando con unos alumnos, había un alumno que faltó y me dice que va a dejar de estudiar porque se va a dedicar a la criptomoneda, se va a dedicar a eso, le deja más, le deja una ganancia de los bitcoins y esa parte, entonces va a dejar de estudiar porque está metido en esa parte. (P.1, esc. 7)

En otra ocasión veo que un alumno se está grabando y le digo: ¿qué haces? Y me dice: es que soy youtuber, maestro, ¿usted sabe cuánto gana un youtuber, maestro? Pues entonces yo quiero ser un youtuber, maestro. (P. 8, esc. 11)

Asimismo, el personal docente refiere que el alumnado presenta falta de claridad en sus intereses y metas; ya que algunos grupos no saben qué es lo que quieren realmente: si dejar el bachillerato para comenzar a trabajar, o continuar estudiando; refieren que trabajando pueden aprovechan más la etapa de la vida por la que atraviesan. Esta afirmación toma sustento en los siguientes comentarios:

Bueno, yo creo que un buen número de estudiantes se pregunta ¿Qué hago? ¿Me pongo a trabajar o continúo mis estudios? Otros no desean hacer ninguna de las dos cosas ya que estudiar representa un esfuerzo importante y trabajar limita su tiempo de paseo y de estar con los amigos. (P.3, esc. 6)

No todos tienen la perspectiva de preparase profesionalmente, de terminar la preparatoria, de terminar la carrera y buscar un trabajo; hay gente que ni siquiera se plantean qué será de su vida o qué quieren lograr; algunos alumnos no saben si realmente están aprovechando su juventud en la escuela cuando podrían estar trabajando. (P. 5, esc. 9)

Como puede verse, desde la perspectiva del profesorado, la falta de un proyecto de vida es un factor que se constituye en barrera para el aprendizaje del estudiantado, lo cual coincide con lo reportado a partir de la información recabada con estudiantes.

Este análisis realizado deja ver la sinergia que existe entre los tres factores estudiados, dado que cada estudiante que posea un autoconcepto positivo, también poseerá metas bien definidas y altas expectativas de vida, elementos que resultan claves en la constitución de un sentido de vida. 
http://doi.org/10.15359/ree.25-1.12

\section{Conclusiones}

Tal como señala la bibliografía en esta investigación, se confirma que una de las principales limitantes del aprendizaje del estudiantado se relaciona con los bloqueos que los grupos mismos erigen, al no saber reconocer sus propias capacidades o hacer una infravaloración de estas; no se sienten capaces de responder a las demandas del nivel educativo. Esta falta de reconocimiento y valoración obedece a una cultura basada en el déficit y no en la capacidad de las personas; es decir, vivimos en una sociedad en donde la retroalimentación de las acciones se centra en señalar los aspectos negativos de las personas que las ejecutan, más que en distinguir los elementos de las tareas que pudieran ser susceptibles de mejora. Este hecho, tal como lo señalan Ibarra Aguirre y Jacobo García (2016), tiene un efecto determinante en los sujetos adolescentes en todos los contextos de su vida y especialmente en el académico.

Es así que, desde la perspectiva del equipo de autoría del presente artículo, la cultura del déficit da cuenta de la dificultad de intervenir en el proceso de construcción de un autoconcepto positivo; ya que esta tarea conlleva un cambio de mentalidad en toda la sociedad (escuela, familia, medios de comunicación, entre otros). No obstante, hay que apostar por lo que se puede hacer desde las instituciones educativas, formando al personal docente tanto en la manera positiva de retroalimentar las actividades escolares, como en el reconocimiento de las potencialidades y de la diversidad presente en las aulas, a fin de que sea capaz de enriquecer el proceso formativo a partir de estos dos factores.

Por otra parte, llama la atención que, aunque las expectativas no se constituyen en una barrera para el aprendizaje del estudiantado en ninguno de los centros educativos, sí existe una relación directa en cuatro de estos, lo cual indica que, a medida que las expectativas disminuyan, también disminuirá el aprendizaje. Este hecho pudiera obedecer a la forma en que se realizó la medición de este factor, en donde únicamente se indagó sobre si el estudiantado consideraba que como consecuencia de concluir el bachillerato o continuar su estudios de nivel superior podrían alcanzar un mejor nivel de vida; pudo ocurrir que sus respuestas obedecieran al valor que la sociedad globalizada ha asignado a la adquisición de una formación profesional, tal como lo refiere la OECD (2019) cuando menciona que cada vez se le otorga un mayor peso a que las personas concluyan su formación profesional como sinónimo de mejor calidad de vida.

Sin embargo, esto no significa que exista consistencia entre las metas que se propone el estudiantado con el esfuerzo y las acciones que está dispuesto a realizar para alcanzarlas; ya que, como refiere el profesorado, estos grupos no tienen la claridad de cómo acercarse a ese horizonte, dado que no son capaces de establecer metas a corto plazo que los acerquen a él.

Respecto al sentido de vida, los resultados obtenidos de la información proporcionada por estudiantes y docentes apunta a que este factor limita el aprendizaje del estudiantado; dado que al no tener claridad en las metas hacia dónde dirigir sus acciones, su compromiso con 
http://doi.org/10.15359/ree.25-1.12

http://www.una.ac.cr/educare

educare@una.ac.cr

las demandas escolares será pobre, y no le encuentra sentido a su aprendizaje. Esta situación evidencia fallas en la implementación de los modelos educativos vigentes en todos los niveles, pues, aunque en estos se declara la búsqueda de una educación integral, la formación para la vida sigue estando infravalorada en favor de la adquisición de conocimientos, tal como lo señala de Barbieri (2018).

Si bien la formación para la vida es una responsabilidad que debe ser compartida por los colectivos que integran las diferentes estructuras de la sociedad (familia, instituciones educativas, instituciones religiosas y medios de comunicación, entre otros), es la escuela la que juega un papel primordial en la definición de un proyecto de vida que permita al alumnado dar sentido a todo lo que hace, idea que coincide con lo propuesto por Bruzzone (2008), quien afirma que es responsabilidad de la escuela ayudar a sus estudiantes a ser mejores personas.

\section{Declaración de Material complementario}

Este artículo tiene disponible, como material complementario:

-La versión preprint del artículo en https://doi.org/10.5281/zenodo.3661198

\section{Referencias}

Abramoski, K., Pierce, J., Hauck, C. y Stoddard, S. (2018). Variations in adolescent purpose in life and their association with lifetime substance use. The Journal of School Nursing, 34(2), 114120. https://doi.org/10.1177/1059840517696964

Booth, T. y Ainscow, M. (2002). Index for inclusion. Developing learning and participation in schools

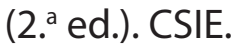

Bruzzone, D. (2008). Pedagogía de las alturas: Logoterapia y educación. LAG.

Calderón, C.y Casu, G. (2011). Escala de factores estructurales de la clase:Una medida para evaluar características de la clase relacionadas con las metas académicas de los estudiantes. Salud \& Sociedad, 2(3), 285-296. https://www.redalyc.org/articulo.oa?id=439742467005

Chacón Borrego, F., Padial Ruz, R., Clements, S. Y., Breton Prats, S., Cepero González, M. y Zurita Ortega, F. (2017). Relación entre el rendimiento académico y autoconcepto en jugadoras de baloncesto de categoría cadete en competición nacional extraescolar. Sport TK: Revista Euroamericana de Ciencias del Deporte, 6(2), 75-80. https://doi.org/10.6018/300411

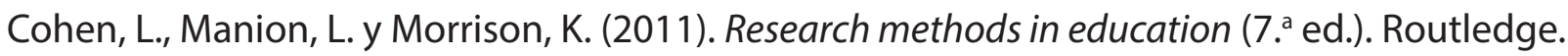


http://doi.org/10.15359/ree.25-1.12

http://www.una.ac.cr/educare educare@una.ac.cr

Costa, S. y Tabernero, C. (2012). Rendimiento académico y autoconcepto en estudiantes de educación secundaria obligatoria según el género. Revista Iberoamericana de Psicología y Salud, 3(2), 175-193. https://www.redalyc.org/pdf/2451/245124456006.pdf

de Barbieri, A. (2018). Educar sin culpa. Optimismo y entusiasmo para padres y docentes. Penguin Randon House Grupo Editorial.

Dirección General de Educación Indígena.(2012). Educación pertinente einclusiva.Ladiscapacidad en educación indígena. Secretaría de Educación Pública. https://www.sep.gob.mx/work/ models/sep1/Resource/8007/1/images/educacion_pertinente e inclusiva.pdf

Frankl, V. (1991). El hombre en búsqueda de sentido. Herder.

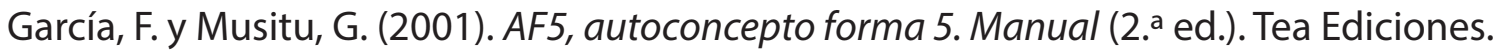

Gámez Rojas, O. (2018). Eficiencia terminal en el sistema de bachillerato del gobierno del Distrito Federal (Tesis de licenciatura). Universidad Pedagógica Nacional Unidad Ajusco. México. http://200.23.113.51/pdf/34126.pdf

Goñi Palacios, E. (2009). El autoconcepto personal: Estructura interna, medida y variabilidad [Tesis de doctorado]. Universidad del País Vasco, España.

Goñi Palacios, E. y Fernández Zabala, A. (2007). Los dominios social y personal del autoconcepto. Revista de Psicodidáctica, 12(2), 179-194. http://www.ehu.eus/ojs/index.php/ psicodidactica/article/viewFile/218/214

Gualtero Pinzón, M. (2016). Importancia del proyecto de vida como eje motivacional para el ingreso a estudios superiores, en los estudiantes de los grados $10^{\circ}$ y $11^{\circ}$ de la institución educativa Técnica La Chamba, del Guamo, Tolima [Tesis de grado]. Universidad de Tolima, Colombia. http://repository.ut.edu.co/bitstream/001/1668/1/IMPORTANCIA\%20DEL\%20 PROYECTO\%20DE\%20VIDA.pdf

Ibarra Aguirre, E. y Jacobo García, H. (2016). La evolución del autoconcepto académico en adolescentes. Revista Mexicana de Investigación Educativa, 21(68), 45-70. http://www. scielo.org.mx/pdf/rmie/v21 n68/1405-6666-rmie-21-68-00045.pdf

Instituto Nacional para la Evaluación de la Educación. (2012). La educación en México: Estado actual y consideraciones sobre su evaluación. http://www.senado.gob.mx/comisiones/ educacion/reu/docs/presentacion 211112.pdf

Kashdam, T. B. y McKnight, P. E. (2009). Origins of purpose in life: Refining our understanding of a life well lived. Psychological Topics, 18(2), 303-316. https://westallen.typepad.com/files/ origins-of-purpose-in-life.pdf 
http://doi.org/10.15359/ree.25-1.12

http://www.una.ac.cr/educare

educare@una.ac.cr

Martín Pavón, M. J. (2015). Optimización del proceso de selección para incrementar la eficiencia terminal en los programas de posgrado [Tesis de doctorado]. Universidad de Granada, España. http://digibug.ugr.es/bitstream/handle/10481/41554/2569697x. pdf? sequence $=1$ \&isAllowed $=y$

Miranda López, F. (2018). Abandono escolar en educación media superior: Conocimiento y aportaciones de política pública. Sinéctica, 51, 1-22. https://doi.org/10.31391/s20077033(2018)0051-010.

Moreno Carmona, M. y Rodríguez Fernández, I. (2010, marzo). Sentido de la vida, inteligencia emocional y salud mental en estudiantes universitarios. En 11a Congreso Virtual de Psiquiatría. Interpsiquis, 11. https://psiquiatria.com/bibliopsiquis/volumen. php?artid $=836 \&$ idrev $=8 \&$ idvol $=137$

Noblejas de la Flor, M. Á. (1994). Logoterapia. Fundamentos, principios y aplicación. Una experiencia de evaluación del "logro interior de sentido" [Tesis de doctorado]. Universidad Complutense de Madrid, España. http://biblioteca.ucm.es/tesis/19911996/S/5/S5005701.pdf

OCDE. (2017). Panorama de la educación 2017. Autor. http://www.oecd.org/education/skillsbeyond-school/EAG2017CN-Mexico-Spanish.pdf

OECD. (2019). Higher Education in Mexico: Labour market relevance and outcomes. Autor. https:// doi.org/10.1787/9789264309432-en

Onrubia Goñi,J. (2009). Transformar para adaptar, adaptar para incluir. Una mirada psicoeducativa a la educación inclusiva. En C. Giné Giné (Coord.), La educación inclusiva. De la exclusión a la plena participación de todo el alumnado (pp. 49-62). Horsori editorial.

Organización de las Naciones Unidas para la Educación, la Ciencia y la Cultura. (2017). Guía para asegurar la inclusión y la equidad en la educación. Autor. https://unesdoc.unesco.org/ ark:/48223/pf0000259592

Papalia, D. E., Wendkos Olds, S. y Duskin Feldman, R. (2009). Desarrollo humano (11. a ed.). McGraw-Hill.

Pereyra López, S. M. y Padilla González, L. E. (2013). Eficiencia terminal en el bachillerato de Aguascalientes. El caso de los centros de estudios científicos y tecnológicos (CECYTEA). XII Congreso Nacional de Investigación Educativa. Aportes y Reflexiones para la Mejora Educativa. Guanajuato, México. http://www.comie.org.mx/congreso/memoriaelectronica/v12/ doc/0327.pdf

Ruiz-Ramírez, R., García-Cué, J. L. y Pérez-Olvera, M. A. (2014). Causas y consecuencias de la deserción escolar en el bachillerato: Caso Universidad Autónoma de Sinaloa. Ra Ximhai, 
http://doi.org/10.15359/ree.25-1.12

http://www.una.ac.cr/educare educare@una.ac.cr

10(5), 51-74. http://www.redalyc.org/articulo.oa?id=46132134004

Sánchez-Sandoval, Y. y Verdugo, L. (2016). Desarrollo y validación de la Escala de expectativas de futuro en la adolescencia. Anales de psicología, 32(2), 545-554. https://doi.org/10.6018/ analesps.32.2.205661

Santana Vega, L. E.y García, L. F.(2011). Percepción de apoyo de padres y profesores, autoconcepto y toma de decisiones en el bachillerato. Revista de Educación, 355, 493-519. 10-4438/1988592X-RE-2011-355-034

Schwinger, M. y Wild, E. (2012). Prevalence, stability, and functionality of achievement goal profiles in mathematics from third to seventh grade. Contemporary Educational Psychology, 37(1), 1-13. https://doi.org/10.1016/j.cedpsych.2011.08.001

Sevilla Santo, D. E., Martín Pavón, M. J., Ramírez de Arellano de la Peña, J. A. y Sunza Chan, S. P. (2020). Barreras personales para el aprendizaje en estudiantes de bachillerato. Contextos educativos, 26, 197-217. https://doi.org/10.18172/con.4227

Sistema de Información de Tendencias Educativas de América Latina. (2010). Metas educativas 2021: Desafíos y oportunidades. Informes sobre tendencias sociales y educativas en América Latina 2010. UNESCO. https://www.google.com.mx/url?sa=t\&rct=j\&q=\&esrc=s\&source= web\&cd=4\&ved=2ahUKEwiF5tGe-ZThAhUMIKwKHbOTC4IQFjADegQIAhAC\&url=https\% 3A\%2F\%2Fwww.oei.es\%2Fhistorico\%2FSITEAL Informe2010.pdf\&usg=AOvVaw26UhNX uoG2mhLIYtIWASFD

Yuen, M., Lee, Q. A. Y., Kam, J. y Lau, P. S. Y. (2017). Purpose in life: A brief review of the literature and its implications for school guidance programs. Journal of Psychologists and Counsellors in Schools, 27(1), 55-69. https://doi.org/10.1017/jgc.2015.18 